\title{
RASTREAMENTO SOCIODEMOGRÁFICO E CLINICO DE INDIVÍDUOS COM DEPRESSÃO
}

\author{
Viviane Gregoleti ${ }^{1}$ \\ Silvana Alba Scortegagna² \\ Marilene Rodrigues Portella ${ }^{3}$
}

resumo

O objetivo deste estudo foi identificar o perfil sociodemográfico e clínico da população diagnosticada com depressão assistida em uma Unidade Básica de Saúde localizada no interior do estado do Rio Grande do Sul, no período de janeiro a dezembro de 2013. Trata-se de um estudo epidemiológico retrospectivo realizado por meio da análise descritiva de prontuários de indivíduos de 5 a 100 anos, caracterizados segundo as variáveis idade, gênero, situação conjugal e medicação utilizada. Identificaram-se 452 prontuários, predominantemente de mulheres (72,1\%), italianas $(55,5 \%) \mathrm{com}$ média de idade de 50,03 anos e casadas (75,5\%). O uso da fluoxetina foi mais significativo entre as mulheres $(p<0,00)$, enquanto 0

1 Graduada em Psicologia. Mestra em Envelhecimento Humano. E-mail: vivigregoleti@yahoo.com.br.

2 Graduada em Psicologia. Doutora em Psicologia. Docente do Programa de Pós-Graduação Stricto Sensu em Envelhecimento Humano da Universidade de Passo Fundo-UPF. E-mail: silvanalba@upf.br. 3 Graduada em Enfermagem. Doutora em Enfermagem. Docente do Programa de Pós-Graduação Stricto Sensu em Envelhecimento Humano da Universidade de Passo Fundo-UPF. E-mail: portella@upf.br. 
diazepam foi mais entre os homens $(p<0,03)$. Os resultados são preocupantes, pois revelam indicadores de depressão acima dos parâmetros mundiais, o que impele o desenvolvimento de ações preventivas e de tratamento à doença nas mulheres, além do uso de medicamentos, e uma reformulação estrutural das questões que compõem os prontuários da unidade.

palavras-chave

Saúde Mental. Atenção Primária à Saúde. Atenção Básica. Epidemiologia.

\section{Introdução}

A depressão é uma das patologias mentais mais frequentes, conferindo o status de mal da contemporaneidade, um importante e crescente problema de saúde pública (BROMET et al., 2011; CUNHA; BASTOS; DEL DUCA, 2012; TEIXEIRA, 2005; PINHEIRO, 2010; GREGOLETI; SCORTEGAGNA, 2015). A doença não surgiu recentemente, pois há mais de 25 séculos o termo era utilizado para designar sintomas ou caracterizar estados mentais, chamada de melancolia, uma condição emocional de infelicidade, desânimo e tristeza (CANALE; FURLAN, 2006; PERES, 2010). No entanto, o que advém atualmente é a democratização da tristeza em sua dimensão mais aguda (PERES, 2010).

Estima-se que até o ano de 2020 a enfermidade alcance o segundo lugar no ranking do cálculo de anos perdidos, ajustados por incapacidade para todas as idades, em ambos os sexos (BLAS; KURUP, 2010; OMS, 2011). Atualmente, a depressão grave é a causa central de incapacitação na população em geral, situando-se em quarto lugar entre as dez principais causas da carga mundial de doenças (BROMET et al., 2011; OMS, 2011).

A doença é caracterizada pela American Psychiatric Association (APA), quinta edição do Manual Diagnóstico e Estatístico de Transtornos Mentais DSM-5 (APA, 2014), pela presença de humor triste, vazio ou irritável, acompanhado de alterações somáticas e cognitivas, que afetam significativamente a capacidade de funcionamento do indivíduo. Esses sintomas diferem quanto ao predomínio e à intensidade, podendo tornar-se crônicos ou recorrentes e levar a substanciais deficiências na capacidade de um indivíduo para cuidar de si mesmo e de suas responsabilidades diárias. Desta forma, a depressão interfere na vida pessoal, profissional, social e econômica, com potencial letal em casos mais graves devido ao risco contínuo de suicídio (SILVA; FUREGATO; 
COSTA JUNIOR, 2003). Quase um milhão de vidas são perdidas anualmente, chegando a atingir um contingente de 3.000 mortes por suicídio todos os dias (WHO, 2012).

No Brasil, a prevalência de depressão na população geral ao longo da vida é de aproximadamente 17\% (ANDRADE et al., 2002; BROMET et al., 2011). Em um estudo realizado em 18 países, a prevalência encontrada foi de $11,1 \%$, e, entre os países de renda média, o maior índice encontrado foi no Brasil, com percentual de 18,4\% (BROMET et al., 2011).

A etiologia da doença é múltipla, fruto de fatores genéticos, bioquímicos, psicológicos e sociofamiliares. Embora possa ocorrer em todas as idades, seus principais preditores são maior faixa etária, gênero feminino, situação conjugal disfuncional, baixos níveis de escolaridade e de renda, condições precárias de moradia, suporte social deficiente e presença de eventos estressores (BROMET et al., 2011; CUNHA; BASTOS; DEL DUCA, 2012; PINHO; CUSTODIO; MAKDISSE, 2009).

Em crianças, devido aos poucos critérios diagnósticos, as taxas de episódios depressivos são baixas, entre 1\% e 3\%, sem diferenciação quanto ao gênero (GOLDMAN, 2012). Entretanto, na adolescência, a depressão encontra-se presente em $49 \%$ a $64 \%$, e é considerada a terceira causa de morte (BEARDSLEE et al., 2012).

Enquanto os índices de prevalência são semelhantes ou mais elevados nos meninos antes da adolescência (ANGOLD; COSTELLO; WORTHMAN, 1998), as meninas apresentam taxas de duas a quatro vezes mais elevadas a partir da adolescência, ao redor de 13 anos de idade, sendo estas associadas mais à puberdade do que à idade cronológica (KESSLER et al., 1997). Essa diferença torna-se ainda mais perceptível entre os 15 e 18 anos de idade, perdurando para o resto da vida (HANKIN et al., 1998). Logo, uma proporção substancial dos transtornos mentais em adultos possui origem na infância ou adolescência (KESSLER et al., 2005; KIM-COHEN et al., 2003).

Em relação aos idosos, a prevalência de sintomas depressivos varia entre $19 \%$ e $34 \%$ nas diferentes regiões do Brasil (BORGES et al., 2013), sendo predominantemente maior em mulheres (GREGOLETI; SCORTEGAGNA, 2015; NOGUEIRA et al., 2014). A etiologia da doença neste segmento da população é complexa, pois há diversos fatores que influenciam o seu surgimento (FUNNELL, 2010). Os mais preponderantes são (a) baixos níveis de escolaridade e socioeconômico, (b) dependência, (c) idade avançada e (d) gênero feminino (GAO et al., 2009; MACIEL; GUERRA, 2006; URBINA TORIJA et al., 2007). Para uma melhor compreensão da doença no cenário brasileiro, nos últimos anos, são demonstrados alguns estudos na Tabela 1. 


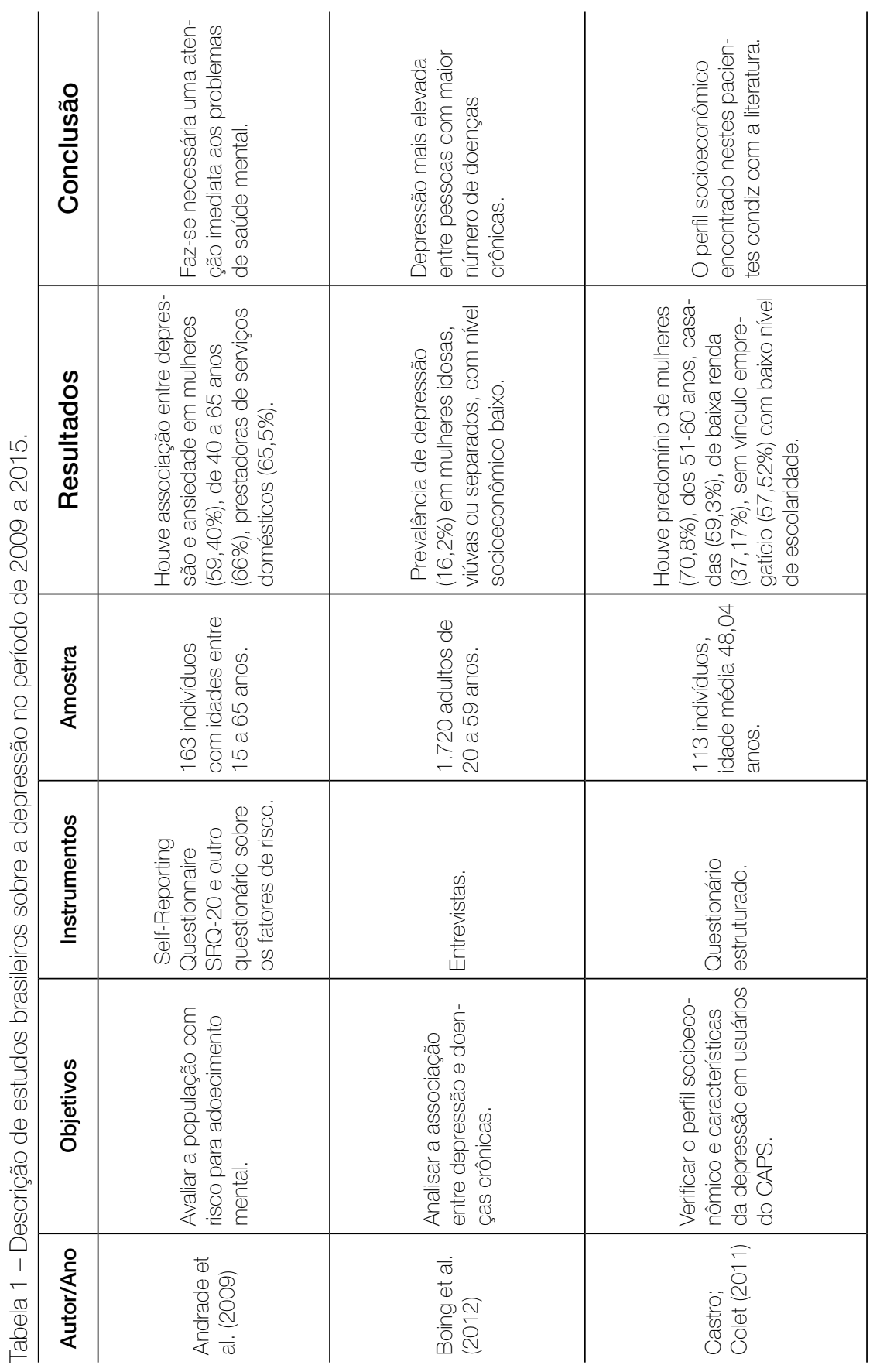

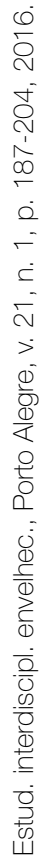




\begin{tabular}{|c|c|c|}
\hline 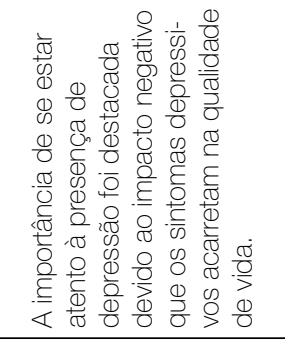 & 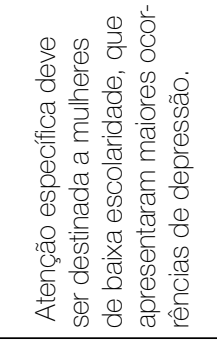 & 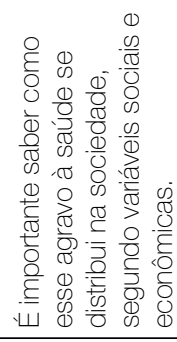 \\
\hline 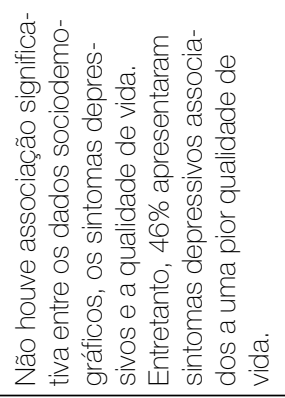 & 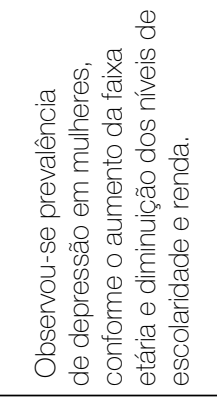 & 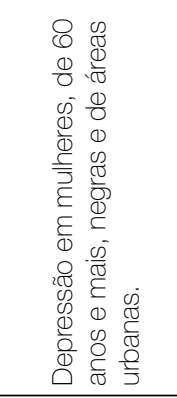 \\
\hline 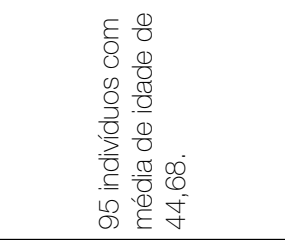 & 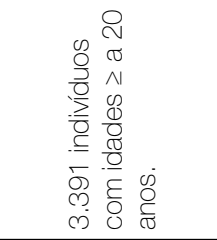 & 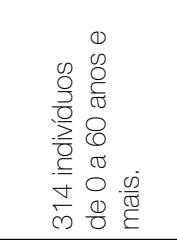 \\
\hline 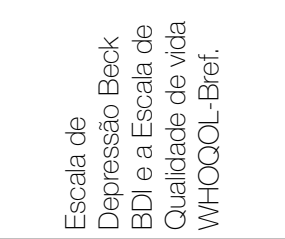 & 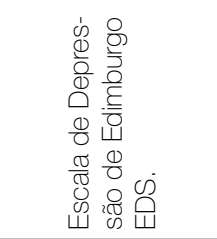 & 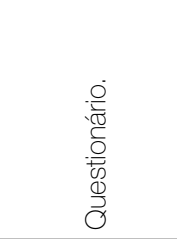 \\
\hline 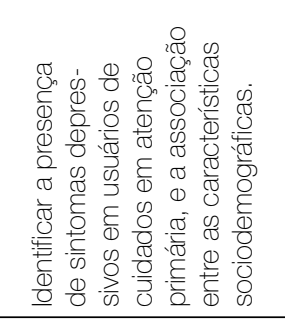 & 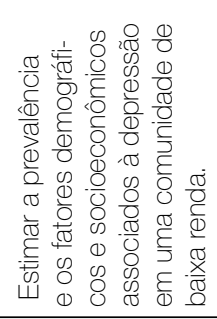 & 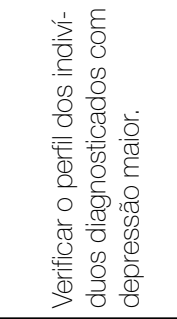 \\
\hline 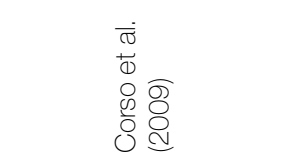 & 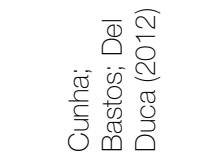 & 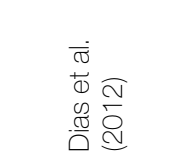 \\
\hline
\end{tabular}

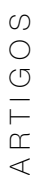

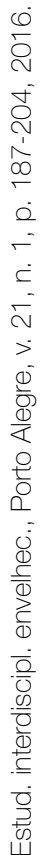


0
0
0
$\square$
$\square$
$\square$

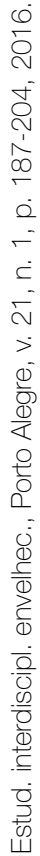

\begin{tabular}{|c|c|c|}
\hline 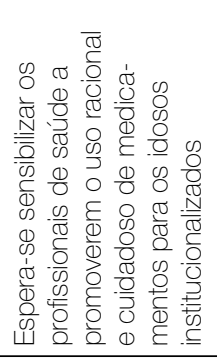 & 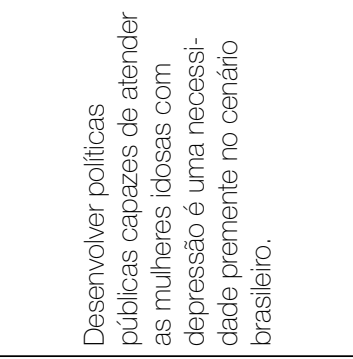 & 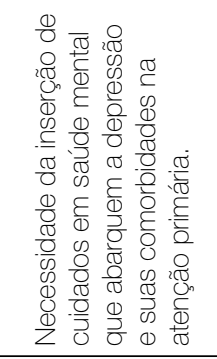 \\
\hline 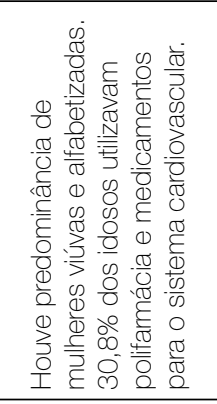 & 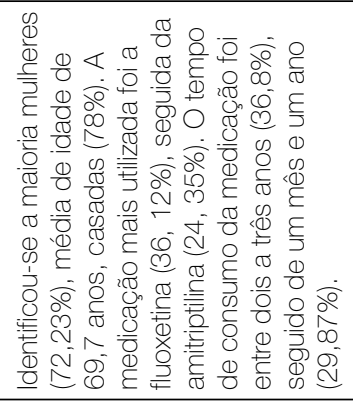 & 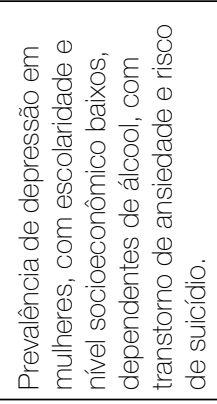 \\
\hline 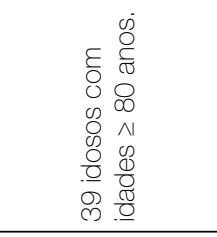 & 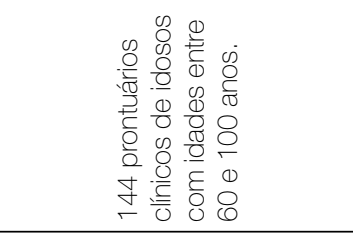 & 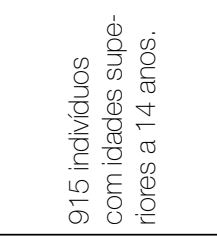 \\
\hline 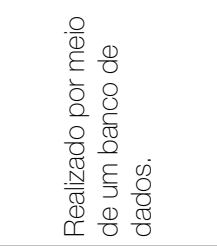 & 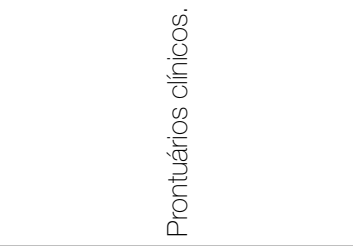 & 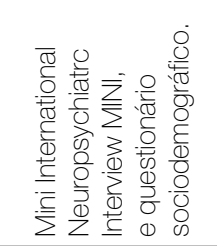 \\
\hline 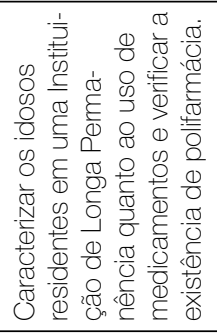 & 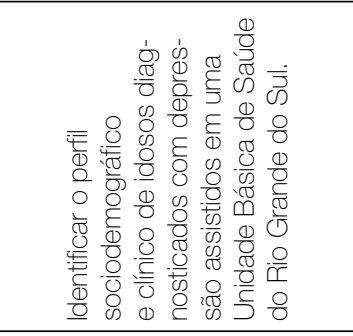 & 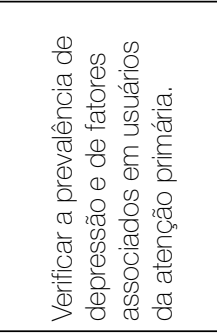 \\
\hline 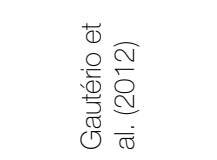 & 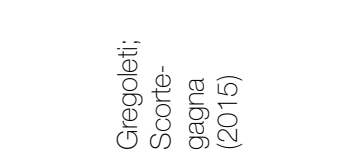 & 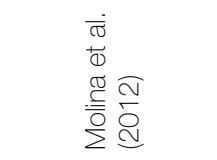 \\
\hline
\end{tabular}




\begin{tabular}{|c|c|c|}
\hline 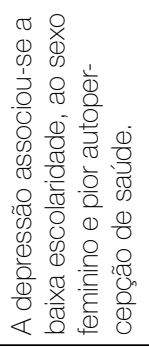 & 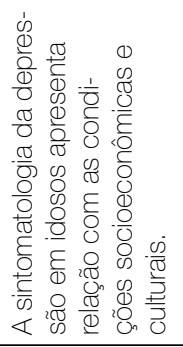 & 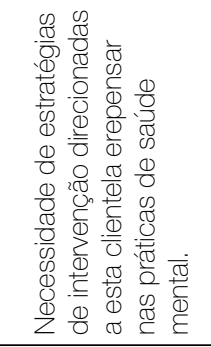 \\
\hline 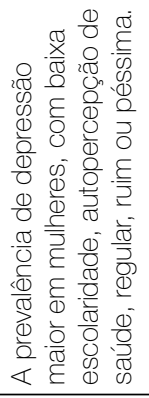 & 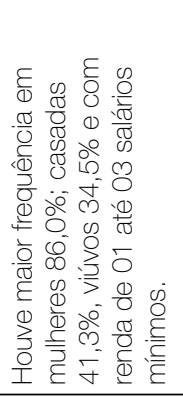 & 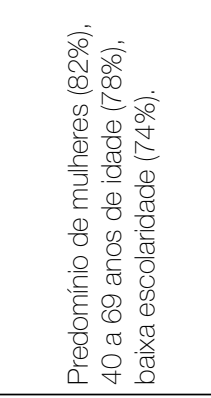 \\
\hline 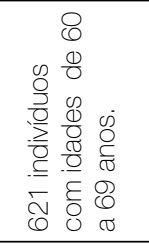 & 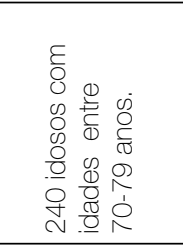 & 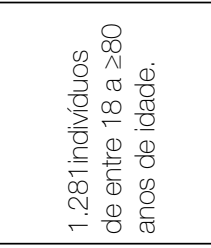 \\
\hline 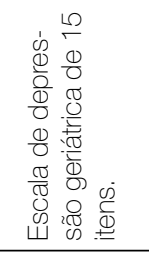 & 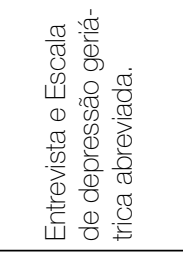 & 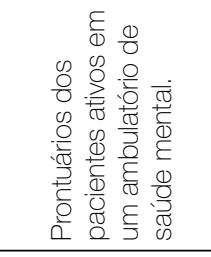 \\
\hline 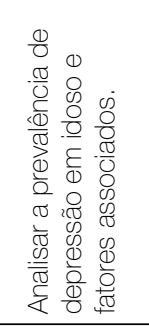 & 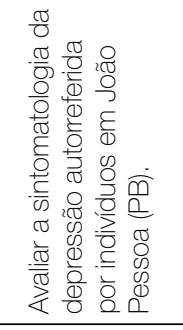 & 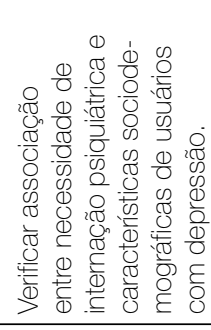 \\
\hline 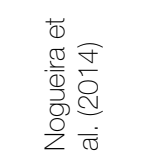 & 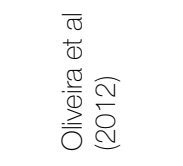 & $\begin{array}{l}\dot{\widetilde{\sigma}} \\
+\frac{\Phi}{\Phi} \\
\frac{\omega}{\Phi} \lesssim \\
\simeq \cong\end{array}$ \\
\hline
\end{tabular}

$\overline{\mathrm{N}}$

$>$

可

4

웅

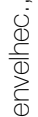

을

竞 
Entre os 12 estudos descritos na Tabela 1, verifica-se a prevalência da psicopatologia em mulheres, em diferentes faixas etárias, com baixa escolaridade e renda. Os principais resultados apontam para a necessidade de estratégias direcionadas à doença e suas comorbidades nesta clientela, na atenção primária (ANDRADE et al., 2009; CUNHA; BASTOS; DEL DUCA, 2012; MOLINA et al., 2012; REIS et al., 2013).

Quanto às modalidades terapêuticas adotadas, os antidepressivos são os medicamentos mais utilizados (ROCHA; WERLANG, 2013). Entre os usuários de antidepressivos de um Centro de Saúde do Município de Nazaré Paulista, de 2003 a 2008, 65,5\% eram do gênero feminino, 40\% casados, 42\% com ensino fundamental incompleto, e 35\% residiam em bairros rurais (SEIXAS et al., 2010).

Outros estudos revelam a prevalência e o padrão de consumo de medicamentos (PEREIRA; FREITAS; QUEIROZ NETTO, 2012; ROCHA; WERLANG, 2013) por usuários do Sistema Único de Saúde SUS, de Ribeirão Preto, SP, e de uma Unidade de Saúde da Família PSF, de Porto Alegre, RS. A distribuição dos usuários de benzodiazepínicos e antidepressivos, identificados no estudo de Pereira, Freitas e Queiroz Netto (2012), destaca a maior prevalência do uso de diazepan entre os homens com faixa etária entre 61 e 70 anos. Entre os utentes de antidepressivos, a fluoxetina e a amitriptilina foram os fármacos mais empregados por mulheres. Em apoio a estes resultados, Rocha e Werlang (2013) demonstram a prevalência de utilização de psicofármacos em mulheres (72\%) com média de idade de 53 anos. Os antidepressivos foram os mais utilizados $(63,2 \%)$, sendo a fluoxetina o medicamento mais consumido $(24,8 \%)$, prosseguido da amitriptilina $(20,4 \%)$.

Como se pode observar, os estudos elencados evidenciam a prevalência de depressão em mulheres, mas a literatura não é conclusiva quanto à faixa etária e à relação da doença com a situação conjugal. Além disso, esta é uma das doenças mais frequentes na atualidade, que causa sofrimento e ocasiona altos custos tanto para o indivíduo como para a saúde pública. Desta forma, é necessário identificar o perfil sociodemográfico e clínico de pessoas diagnosticadas com depressão na atenção primária. 
Trata-se de um estudo epidemiológico, retrospectivo, realizado por meio da análise dos prontuários dos pacientes com depressão, com idades entre 5 a 100 anos, provenientes de uma Unidade Básica de Saúde UBS, de Água Santa, Rio Grande do Sul. A USB possui os dados epidemiológicos de todo o município, que possui uma população residente de 3.722 habitantes, 1.879 do gênero masculino e 1.843 do gênero feminino (IBGE, 2010). O diagnóstico de depressão, presente nos prontuários, foi realizado pelo médico da Estratégia Saúde da Família ESF, com base nas diretrizes da Classificação Internacional de Doenças (CID-10).

A coleta de dados abrangeu o período de 01/01/2013 a 31/12/2013, totalizando 12 meses, e foi realizada nas dependências da UBS. Para atender ao objetivo proposto, os dados dos prontuários foram caracterizados segundo as variáveis idade, gênero, situação conjugal, etnia, e medicações utilizadas. Outras variáveis como escolaridade, nível socioeconômico e condições familiares não foram encontrados registros. O levantamento e a análise dos dados contaram com a estatística descritiva de corte transversal. Para tanto, utilizou-se o Statistical Package for the Social Sciences SPSS, versão 18.0, e realizou-se o teste de Pearson. O nível de significância considerado foi $\alpha=0,05$. O projeto foi aprovado pelo Comitê de Ética em Pesquisa da Universidade de Passo fundo, sob o parecer de número 169.507, emitido em 12/12/2012, cumprindo-se, assim, as exigências da Resolução n. - 466/12 do Conselho Nacional de Saúde.

\section{Resultados}

Em um período de 12 meses, entre janeiro a dezembro de 2013, foram localizados 452 prontuários. Os dados de caracterização relativos a gênero, idade, situação conjugal, etnia e número de medicamentos utilizados encontram-se descritos na Tabela 2. 
Tabela 2 - Descrição das variáveis sociodemográficas e clínicas de indivíduos com depressão.

\begin{tabular}{|c|c|c|c|c|c|}
\hline \multirow{2}{*}{ Variáveis } & & \multicolumn{2}{|c|}{ Masculino } & \multicolumn{2}{|c|}{ Feminino } \\
\hline & & $\mathrm{n}$ & $\%$ & $\mathrm{n}$ & $\%$ \\
\hline Gênero & & 126 & 27,9 & 326 & 72,1 \\
\hline \multirow{5}{*}{ Idade } & $5-12$ & 7 & 5,6 & 1 & 0,3 \\
\hline & $13-19$ & 3 & 2,4 & 15 & 4,6 \\
\hline & $20-39$ & 21 & 16,7 & 66 & 20,2 \\
\hline & $40-59$ & 58 & 46 & 147 & 45,1 \\
\hline & $>$ de 60 anos & 37 & 29,4 & 97 & 29,8 \\
\hline \multirow{4}{*}{$\begin{array}{l}\text { Situação } \\
\text { Conjugal }\end{array}$} & Casado (a) & 90 & 71,4 & 246 & 75,5 \\
\hline & Solteiro (a) & 29 & 23 & 37 & 11,3 \\
\hline & Separado (a) & 4 & 3,2 & 16 & 4,9 \\
\hline & Viúvo (a) & 3 & 2,4 & 27 & 8,3 \\
\hline \multirow{4}{*}{ Etnia } & Italiano (a) & 79 & 62,7 & 182 & 55,8 \\
\hline & Brasileiro (a) & 42 & 33,3 & 122 & 37,4 \\
\hline & Alemão (a) & 4 & 3,2 & 16 & 4,9 \\
\hline & Indígena & 1 & 0,8 & 6 & 1,8 \\
\hline \multirow{5}{*}{$\begin{array}{l}\text { Número de } \\
\text { Medicamentos }\end{array}$} & 1 & 97 & 77 & 239 & 73,3 \\
\hline & 2 & 23 & 18,3 & 65 & 19,9 \\
\hline & 3 & 4 & 3,2 & 17 & 5,2 \\
\hline & 4 & 2 & 1,6 & 4 & 1,2 \\
\hline & 5 & 0 & 0 & 1 & 0,5 \\
\hline
\end{tabular}

Fonte: Tabela elaborada pelas autoras

Observa-se na Tabela 2 que 72,1\% dos indivíduos diagnosticados com depressão foram mulheres, com uma média de idade de 50,03 anos, casadas (75,5\%), e de origem étnica italiana (55,8\%). Outros resultados revelam que a doença foi preponderante entre os meninos com até 12 anos de idade $(5,6 \%)$ em comparação com as meninas $(0,3 \%)$. Já nos adolescentes, as meninas $(4,6 \%)$ apresentaram mais diagnóstico da enfermidade que os rapazes $(2,4 \%)$.

A classe de antidepressivos, considerada um dos recursos utilizados para o tratamento da depressão nas USB, compreende: a fluoxetina, a amitriptilina, 
o carbonato de lítio, o diazepan, o clonazepan, a imipramina e a nortriptilina. Na Tabela 3, encontram-se descritos os antidepressivos empregados por ambos os gêneros.

Tabela 3 - Descrição dos antidepressivos utilizados por indivíduos com depressão de acordo com o gênero.

\begin{tabular}{l|c|c|c|c}
\hline \multicolumn{1}{c|}{ Medicação } & Gênero & $\begin{array}{c}\text { Sim } \\
\mathbf{n}(\%)\end{array}$ & $\begin{array}{c}\text { Não } \\
\mathbf{n}(\%)\end{array}$ & P \\
\hline \multirow{2}{*}{ Fluoxetina } & Masculino & $47(37,3 \%)$ & $79(62,7 \%)$ & 0,00 \\
\cline { 2 - 5 } & Feminino & $167(51,2 \%)$ & $159(48,8 \%)$ & \\
\hline \multirow{3}{*}{ Amitriptilina } & Masculino & $37(29,4 \%)$ & $89(70,6 \%)$ & 0,90 \\
\cline { 2 - 5 } & Feminino & $95(29,1 \%)$ & $231(70,9 \%)$ & \\
\hline \multirow{3}{*}{ Carbonato de Lítio } & Masculino & $9(7,1 \%)$ & $117(92,9 \%)$ & 0,10 \\
\cline { 2 - 5 } & Feminino & $12(3,7 \%)$ & $314(96,3 \%)$ & \\
\hline \multirow{2}{*}{ Diazepam } & Masculino & $26(20,6 \%)$ & $100(79,4 \%)$ & 0,03 \\
\cline { 2 - 5 } & Feminino & $41(12,6 \%)$ & $285(87,4 \%)$ & \\
\hline \multirow{2}{*}{ Imipramina } & Masculino & $34(27 \%)$ & $92(73 \%)$ & 0,80 \\
\cline { 2 - 5 } & Feminino & $86(26,4 \%)$ & $240(73,6 \%)$ & \\
\hline \multirow{2}{*}{ Nortriptilina } & Masculino & $10(7,9 \%)$ & $116(92,1 \%)$ & 0,60 \\
\cline { 2 - 5 } & Feminino & $30(9,2 \%)$ & $296(90,8 \%)$ & \\
\hline
\end{tabular}

Fonte: Tabela elaborada pelas autoras

Pode se verificar na Tabela 3 que a medicação mais utilizada foi a fluoxetina tanto para as mulheres $(51,2 \%)$ como para os homens $(37,3 \%)$. Entretanto, as mulheres foram as que mais utilizaram essa medicação, com nível de significância importante $(\mathrm{p}<0,00)$. Para os homens, o diazepam foi o mais significativo $(p<0,03)$. As demais medicações, mesmo não havendo nível de significância importante, foram utilizadas por um número expressivo de indivíduos, a exemplo, a amitriptilina, seguida do clonazepam. 
Este estudo permitiu identificar o perfil sociodemográfico e clínico da população de uma cidade do interior do estado do RS diagnosticada com depressão e assistida em uma USB. As variáveis observadas demonstram que as mulheres casadas têm mais depressão do que os homens. Essas características assemelham-se às tendências observadas nos estudos populacionais brasileiros (ANDRADE et al., 2009; BOING et al., 2012; CASTRO; COLET, 2011; CUNHA; BASTOS; DEL DUCA, 2013; MOLINA et al., 2012; OLIVEIRA et al., 2012; REIS et al., 2013; SEIXAS et al., 2010) e com a inclusão de amostras brasileiras (BROMET et al., 2011).

Embora a depressão seja a principal causa de incapacidade para ambos os sexos, as mulheres possuem $50 \%$ mais chances de desenvolver depressão do que homens (WHO, 2012). A preocupação com a maior incidência de depressão em mulheres é também ressaltada por outros autores (ANDRADE et al., 2009; BOING et al., 2012; BROMET et al., 2011; CASTRO; COLET, 2011; CORSO et al., 2009; CUNHA; BASTOS; DEL DUCCA, 2013; GAUTÉRIO et al., 2012; GREGOLETI; SCORTEGAGNA, 2015; MOLINA et al., 2012; OLIVEIRA et al., 2012; REIS et al., 2013).

Contudo, ressalta-se que considerando a população total do município pesquisado de 3.722 habitantes, 422 indivíduos (12\%) possuem diagnóstico de depressão. Agrava-se, ainda mais, a prevalência de doença em mulheres, quase três vezes mais do que em homens. Residem no município 1.843 mulheres, sendo diagnosticadas com depressão 326, o que condiz com $17,68 \%$ da população feminina. Enquanto que os homens perfazem um total de 1.879 habitantes, sendo diagnosticados com depressão 126, o que resulta em 6,7\% da população masculina.

De maneira geral, são vários os fatores apontados para que este fenômeno ocorra com maior frequência nas mulheres, entre eles as questões biológicas, principalmente as alterações hormonais (SOARES; ZITEK, 2008; LOPEZ et al., 2011). Outros autores apontam a influência das questões socioculturais e psicológicas, bem como a maior vulnerabilidade a eventos estressantes como casamentos insatisfatórios, abandono por parte de seus companheiros e cuidado com parentes doentes ou incapacitados (CUNHA; BASTOS; DEL DUCA, 2012). Outro fator referido é que as mulheres procuram mais os serviços de saúde (SEIXAS et al., 2010), pois cuidam-se mais do que os homens (ROCHA; WERLANG, 2013).

Os adultos mais velhos mostram uma tendência mais elevada para o desenvolvimento de depressão do que os adultos mais jovens (ANDRADE et al., 2009; BOING et al., 2012). Esses achados corroboram com os resultados obtidos 
neste estudo, em que a maior parte dos indivíduos com depressão encontra-se na faixa etária entre os 41 e 60 anos. Este achado pode estar associado a questões hormonais como a menopausa (SOARES; ZITEK, 2008; LOPEZ et al., 2011), à aposentadoria, que, muitas vezes, é permeada pela perda econômica e do status social e familiar, especialmente na cultura ocidental, à consciência da finitude que se amplia, à emergência de doenças crônicas e às perdas de entes queridos (GREGOLETI; SCORTEGAGNA, 2015).

O consumo de mais medicações para a depressão em mulheres do que em homens foi evidenciado em outras pesquisas (GREGOLETI; SCORTEGAGNA, 2015; SEIXAS et al., 2010; ROCHA; WERLANG, 2013). De modo similar, Rabelo e Tavares (2008) mostraram a associação entre sofrimento e relações socioafetivas com o consumo de medicamentos. Ou seja, a subordinação de muitas mulheres aos maridos, aos conflitos conjugais, à violência de gênero e à sobrecarga dos cuidados familiares, faz com que recorram aos serviços de saúde visando aplacar seu sofrimento, tendo por retorno a medicalização. Essas evidências apoiam-se nos achados que mostram que a maior parte da população com depressão é casada (CASTRO; COLET, 2011; CORSO et al., 2009; CUNHA; BASTOS; DEL DUCA, 2012; OLIVEIRA et al., 2012; SEIXAS et al., 2010; GREGOLETI; SCORTEGAGNA, 2015), o que representa 75,5\% das mulheres deste estudo, uma condição que precisa ser melhor explorada.

Outros resultados revelam que a maioria das crianças com até 12 anos de idade com depressão são meninos $(5,6 \%)$, o que foi confirmado na literatura prévia (ANGOLD; COSTELLO; WORTHMAN, 1998). Já nos adolescentes, as meninas $(4,6 \%)$ possuem mais depressão que os meninos $(2,4 \%)$, validando estudos anteriores (HANKIN et al., 1998; KESSLER et al., 1997; MARCOTTE; LEMIEUX, 2014).

É importante destacar que certos jovens são mais vulneráveis à depressão e nem todos seguem a mesma trajetória. Embora a população dos rapazes não costume apresentar um aumento significativo de sintomas depressivos quando da transição para a adolescência, a partir de 11-12 anos de idade ou no sexto ano do ensino fundamental, os que vivem uma puberdade precoce associada a uma imagem corporal negativa, que possuem conflitos familiares e não recebem apoio dos amigos próximos, dos quais mantêm certa dependência afetiva, são mais vulneráveis à depressão. Nas mulheres, os desafios normativos da adolescência podem aumentar a vulnerabilidade e influenciar o risco à depressão: a ruptura dos laços familiares e sociais infantis, uma imagem corporal negativa quando associada a dificuldades familiares e a transição do ensino fundamental para o ensino médio são fatores a serem considerados na emergência do quadro (MARCOTTE; LEMIEUX, 2014). 
Quanto às modalidades terapêuticas, a fluoxetina foi o medicamento mais utilizado entre as mulheres e os homens, o que também foi reportado em outras pesquisas (ROCHA; WERLANG, 2013; SEIXAS et al., 2010). As mulheres em diferentes faixas etárias são as que mais utilizam essa medicação, notadamente as idosas (GREGOLETI; SCORTEGAGNA, 2015; ROCHA; WERLANG, 2013) enquanto o uso de diazepam é mais significativo entre os homens, inclusive os idosos (FIRMINO et al., 2011; PEREIRA; FREITAS; QUEIROZ NETTO, 2012). $\mathrm{O}$ uso desses medicamentos pode ser devido à medicalização da sociedade, às pressões mercadológicas da indústria farmacêutica e ao envelhecimento (PEREIRA; FREITAS; QUEIROZ NETTO, 2012).

O diazepam é um dos benzodiazepínicos mais antigos e conhecidos pela população, e seu uso tem como objetivo contornar efeitos adversos, particularmente a insônia, de outras classes de psicotrópicos, como o caso dos antidepressivos (FIRMINO et al., 2011). De qualquer modo, deve-se destacar que os fatores que geram prevalência da utilização do diazepam em homens não são claros e devem ser mais bem investigados. Corroborando com estes resultados, o estudo prévio de Chaimowicz Ferreira e Miguel (2000) mostra que o consumo desses medicamentos está relacionado ao fato do envelhecimento muitas vezes ser acompanhado pelo aparecimento de transtornos do sono, depressão e doenças neurológicas degenerativas. Entretanto, Pereira, Freitas e Queiroz Netto (2012) alertam que a maior utilização de benzodiazepínicos em pacientes com faixas etárias mais elevadas apresenta-se inadequada, em especial aqueles de meia vida longa, como os comumente distribuídos em UBS, já que produzem sedação prolongada, o que pode aumentar o risco de quedas e de fraturas.

\section{Conclusão}

Desses resultados, derivam vários desdobramentos que podem contribuir para o direcionamento de programas de saúde mental, especialmente para a população feminina deste estudo. Considerando que a meta do tratamento não deve ser apenas a recuperação da doença, mas a prevenção tanto da sua recorrência como da emergência de novos casos, destacam-se: a) a necessidade do desenvolvimento de grupos de apoio aos pacientes com o objetivo de auxiliar no resgate de uma imagem corporal positiva, de uma boa autoestima; na resolução de problemas interpessoais e de conflitos familiares; e na promoção de habilidades sociais; b) o desenvolvimento de oficinas dirigidas à comunidade, 
com vistas a ampliar o conhecimento sobre a depressão e estimular a auto-observação; e c) a urgência de uma reformulação estrutural das questões que compõem os prontuários da USB.

Embora este estudo tenha trazido contribuições no sentido de conhecer a população do município com depressão e auxiliar o direcionamento de ações, ao menos duas limitações devem ser consideradas. A primeira diz respeito à falta de informação acerca de outras variáveis sociodemográficas e de saúde como escolaridade, nível socioeconômico, e condições familiares, ausentes nos prontuários clínicos, o que impossibilitou uma maior abrangência dos achados. A segunda refere-se ao fato do diagnóstico ter sido obtido por meio da revisão dos prontuários médicos dos pacientes. Há de se considerar que estudos prospectivos, controlados, de longa duração e que visem à detecção e ao acompanhamento da população com depressão sejam imprescindíveis e poderiam fornecer dados definitivos.

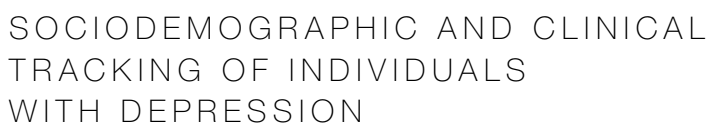

\section{abstract}

The aim of this study was to identify the sociodemographic and clinical profile of the population diagnosed with depression assisted in a Basic Health Unit, located in the state of Rio Grande do Sul, in the period from January to December of 2013. This is a retrospective epidemiological study done through a descriptive analysis of individuals' records from 5 to 100 years, characterized according to their age, gender, marital status, used medication. Were identified 452 records, predominantly of women (72.1\%), Italian (55.5\%) with a mean age of 50.03 years, married (75.5\%). The use of fluoxetine was the most significant among women $(\mathrm{p}<0.00)$, whereas diazepam among men $(p<0.03)$. The results are worrying, because they reveal indicators of depression over the global parameters what drives the development of preventive actions and of a treatment to the disease in women, beyond the use of medicines, and a structural reformulation of the questions that compose the records of the unit.

keywords

Mental Health. Primary Attention to Health. Basic Attention. Epidemiology. 
AMERICAN PSYCHIATRIC ASSOCIATION. Manual Diagnóstico e Estatístico de Transtornos Mentais: DSM-5. 5. ed. Porto Alegre: Artmed, 2014.

ANDRADE, Fábia Barbosa et al. Saúde mental na atenção básica: um estudo epidemiológico baseado no enfoque de risco. Revista Brasileira de Enfermagem, Brasília, v. 62, n. 5, p. 675-689, set./out. 2009.

ANDRADE, Laura et al. Prevalence of ICD-10 mental disorders in a catchment area in the city of São Paulo, Brazil. Social Psychiatry and Psychiatric Epidemiology, Berlin, v. 37, n. 7, p. 316-325, July 2002.

ANGOLD, Adrian; COSTELLO, Elizabeth Jane; WORTHMAN, Carol M. Puberty and depression: The roles of age, pubertal status and pubertal timing. Psychological Medicine, London, v. 28, n. 1, p. 51-61, Jan. 1998.

BEARDSLEE, William R.; GLADSTONE, Tracy R. G.; O'CONNOR, Erin E. Developmental risk of depression: experience matters. Child and Adolescent Psychiatric Clinics of North America, Philadelphia, PA, v. 21, n. 2, p. 261-278, Apr. 2012.

BLAS, Erik; KURUP, Anand Sivasankara (Ed.). Equity, social determinants and public health programmes. Geneva: World Health Organization, 2010. Disponivel em: <http://whqlibdoc.who.int/publications/2010/9789241563970_eng.pdf>. Acesso em: 28 fev. 2016.

BOING, Antonio Fernando et al. Associação entre depressão e doenças crônicas: um estudo populacional. Revista Saúde Pública, São Paulo, v. 46, n. 4, p. 617-623, ago. 2012.

BORGES, Lucélia Justino et al. Fatores associados aos sintomas depressivos em idosos: estudo EpiFloripa. Revista Saúde Pública, São Paulo, v. 47, n. 4, p. 701-710, ago. 2013

BROMET, Evelyn et al. Cross-national epidemiology of DSM-IV major depressive episode. BMC Medicine, London, v. 9, n. 90, p. 1-16, July 2011.

CANALE, Alaíse; FURLAN, Maria Montserrat Diaz Pedrosa. Depressão. Arquivos do MUDI, Maringá, v. 10, n. 2, p. 23-31, 2006.

CASTRO, Ana Luiza Ferreira Meinen de; COLET, Christiane de Fátima. Perfil socioeconômico e características da depressão de usuários do Centro de Atenção Psicossocial (CAPS) de Panambi/RS. Revista Contexto \& Saúde, ljuí, v. 11, n. 20, p. 401-408, 2011.

CHAIMOWICZ, Flávio; FERREIRA, Teresinha de Jesus Xavier Martins; MIGUEL, Denise Freire Assumpção. Uso de medicamentos psicoativos e seu relacionamento com quedas entre idosos. Revista Saúde Pública, São Paulo, v. 34, n. 6, p. 631-635, dez. 2000.

CORSO, Aline Naymayer et al. Impacto de sintomas depressivos na qualidade de vida de usuários da rede básica de saúde. Revista Gaúcha de Enfermagem, Porto Alegre, v. 30, n. 2, p. 257-262, jun. 2009.

CUNHA, Ricardo Vivian da; BASTOS, Gisele Alsina Nader; DEL DUCA, Giovâni Firpo. Prevalência de depressão e fatores associados em comunidade de baixa renda de Porto Alegre, Rio Grande do Sul. Revista Brasileira de Epidemiologia, São Paulo, v. 15, n. 2, p. 346-354, jun. 2012.

FIRMINO, Karleyla Fassarela et al. Fatores associados ao uso de benzodiazepínicos no serviço municipal de saúde da cidade de Coronel Fabriciano, Minas Gerais, Brasil. Cadernos de Saúde Pública, Rio de Janeiro, v. 27, n. 6, p. 1223-1232, jun. 2011.

FUNNELL, Emma. Depression in the elderly. InnovAiT, Liverpool, v. 3, n. 4, p. 199-208, Apr. 2010. 
GAO, Sujuan et al. Correlates of depressive symptoms in rural elderly Chinese. International Journal of Geriatric Psychiatry, Chichester, v. 24, n. 12, p. 1358- 1366, dez. 2009

GAUTÉRIO, Daiane Porto et al. Caracterização dos idosos usuários de medicação residentes em instituição de longa permanência. Revista da Escola de Enfermagem da USP, São Paulo, v. 46, n. 6, p. 1394-1399, dez. 2012.

GOLDMAN, Stuart. Developmental epidemiology of depressive disorders. Child and Adolescent Psychiatric Clinics of North America, Philadelphia, PA, v. 21, n. 2, p. 217-235, Apr. 2012

GREGOLETI, Viviane; SCORTEGAGNA, Silvana Alba. Perfil sociodemográfico e clínico da população de idosos com transtorno depressivo. Estudos Interdisciplinares sobre o Envelhecimento, Porto Alegre, v. 20, n. 1, p. 271-283, abr. 2015.

HANKIN, Benjamin L. et al. Development of depression from a preadolescence to young adulthood: Emerging gender differences in a 10-year longitudinal study. Journal of Abnormal Psychology, Washington, D.C., v. 107, n. 1, p. 128-140, Feb. 1998.

INSTITUTO BRASILEIRO DE GEOGRAFIA E ESTATÍSTICA. Cidades. Água Santa, 2010. Disponível em: <http://www.cidades.ibge.gov.br/xtras/temas.php?lang=\& codmun $=430005 \&$ idtema=16\&search=rio-grande-do-sul|\%C3\%81gua-santa|sintesedas-informacoes>. Acesso em: 20 fev. 2016.

KESSLER, Ronald C. et al. Prevalence, correlates, and course of minor depression and major depression in the national comorbidity survey. Journal of Affective Disorders, Amsterdam, v. 45, n. 1-2, p. 19-30, Aug. 1997.

KESSLER, Ronald C. et al. Lifetime prevalence and age-of-onset distributions of DSM-IV disorders in the National Comorbidity Survey Replication. Archives of General Psychiatry, Chicago, v. 62, n. 6, p.593-602, June 2005.

KIM-COHEN, Julia et al. Prior Juvenile Diagnoses in Adults With Mental Disorder: Developmental Follow-Back of a Prospective-Longitudinal Cohort. Archives of General Psychiatry, Chicago, v. 60, n. 7, p. 709-717, July 2003.

LOPEZ, Mariane Ricardo Acosta et al. Depressão e qualidade de vida em jovens de 18 a 24 anos no sul do Brasil. Revista de Psiquiatria do Rio Grande do Sul, Porto Alegre, v. 33, n. 2, p. 103-108, abr. 2011

MACIEL, Álvaro Campos Cavalcanti; GUERRA, Ricardo Oliveira. Prevalência e fatores associados à sintomatologia depressiva em idosos residentes no Nordeste do Brasil. Jornal Brasileiro de Psiquiatria, Rio de Janeiro, v. 55, n. 1, p. 26-33, 2006.

MARCOTTE, Diane; LEMIEUX, Annie. La trajectoire des symptômes dépressifs de l'enfance à l'adolescence et les cibles d'intervention préventive. Ciência \& Saúde Coletiva, Rio de Janeiro, v. 19, n. 3, p. 785-796, mars 2014.

MOLINA, Mariane Ricardo Acosta Lopez et al. Prevalência de depressão em usuários de unidades de atenção primária. Revista de Psiquiatria Clínica, São Paulo, v. 39, n. 6, p. 194-197, 2012.

NOGUEIRA, Eduardo Lopes et al. Rastreamento de sintomas depressivos em idosos na Estratégia Saúde da Família, Porto Alegre. Revista Saúde Pública, São Paulo, v. 48, n. 3, p. 368-377, jun. 2014

OLIVEIRA, Marcos Francisco de et al. Sintomatologia de depressão autorreferida por idosos que vivem em comunidade. Ciência \& Saúde Coletiva, Rio de Janeiro, v. 17, n. 8, p. 2191-2198, ago. 2012.

ORGANIZACIÓN MUNDIAL DE LA SALUD. Estadísticas sanitarias mundiales 2011. Ginebra: OMS, 2011. Disponível em: <http://www.who.int/gho/publications/world_ health_statistics/ES_WHS2011_Full.pdf>. Acesso em: 28 fev. 2016. 
ORGANIZAÇÃO MUNDIAL DA SAÚDE. Classificação Estatística Internacional de Doenças e Problemas Relacionados à Saúde-Décima Revisão. 8. ed. São Paulo: Edusp, 2008. v. 2

PEREIRA, Leonardo Régis Leira; FREITAS, Osvaldo; QUEIROZ NETTO, Maira Umezaki. Antidepressivos e Benzodiazepínicos: estudo sobre o uso racional entre usuários do SUS em Ribeirão Preto-SP. Revista de Ciências Farmacêuticas Básica e Aplicada, Araraquara, v. 33, n. 1, p. 77-81, 2012.

PERES, Urania Tourinho. Depressão e Melancolia. 3. ed. Rio de Janeiro: Jorge Zahar Editora, 2010

PINHEIRO, Maria Teresa da Silveira; QUINTELLA, Rogerio Robbe; VERZTMAN, Julio Sergio. Distinção teórico-clínica entre depressão, luto e melancolia. Psicologia Clínica, Rio de Janeiro, v. 22, n. 2, p. 147-168, 2010

PINHO, Miriam Ximenes; CUSTODIO, Osvladir; MAKDISSE, Marcia. Incidência de depressão e fatores associados em idosos residentes na comunidade: revisão de literatura. Revista Brasileira de Geriatria e Gerontologia, Rio de Janeiro, v. 12, n. 1 , p. 123-140, jan./abr. 2009.

RABELO, Ionara Vieira Moura, TAVARES, Rosana Carneiro. Homens-carrapatos e suas mulheres: relato de experiência em Saúde Mental na Estratégia Saúde da Família. Saúde em Debate, Rio de Janeiro, v. 32, n. 78/79/80, p. 133-142, jan./dez. 2008.

REIS, Leonardo Naves et al. Probabilidade de internação psiquiátrica e características sociodemográficas de portadores de depressão. Revista Eletrônica de Enfermagem, Goiânia, v. 15, n. 4, p. 862-869, out./dez. 2013.

ROCHA, Bruno Simas da; WERLANG, Maria Cristina. Psicofármacos na Estratégia Saúde da Família: perfil de utilização, acesso e estratégias para a promoção do uso racional. Ciência \& Saúde Coletiva, Rio de Janeiro, v. 18, n. 11, p. 3291-3300, nov. 2013.

SEIXAS, Sônia Regina da Cal et al. Diagnósticos e medicalização: algumas considerações sobre depressão e subjetividade em Nazaré Paulista, Apa Cantareira, São Paulo, Brasil. Mudanças - Psicologia da Saúde, São Paulo, v. 18, n. 1-2, p. 7-19, jan./dez. 2010.

SILVA, Mariluci Camargo Ferreira da; FUREGATO, Antonia Regina Ferreira; COSTA JúNIOR, Moacyr Lobo da Costa. Depressão: pontos de vista e conhecimento de enfermeiros da rede básica de saúde. Revista Latino-Americana de Enfermagem, Ribeirão Preto, v. 11, n. 1, p. 7-13, jan./fev. 2003

SOARES, Claudio N.; ZITEK, Brook. Reproductive hormone sensitivity and risk for depression across the female life cycle: a continuum of vulnerability? Journal of Psychiatry \& Neuroscience, Ottawa, v. 33, n. 4, p. 331-343, July 2008

TEIXEIRA, Marco Antônio Rotta. Melancolia e depressão: um resgate histórico e conceitual na psicanálise e na psiquiatria. Revista de Psicologia da UNESP, São Paulo, v. 4, n. 1, p. 41-56, 2005

URBINA TORIJA, Juan Román et al. Síntomas depresivos en personas mayores. Prevalencia y factores asociados. Gaceta Sanitaria, Barcelona, v. 21, n. 1, p. 37-42, enero/feb. 2007.

WORLDS HEALTH ORGANIZATION .World suicide, prevention day. Geneva: WHO, 2012. Disponível em: <http://www.who.int/mediacentre/events/annual/world_ suicide_prevention_day/en/>. Acesso em: 28 fev. 2016. 